\title{
Accuracy of Distal Long Femur Nail Locking with Different Techniques
}

\author{
Ozgur Basal*1, Vecihi Kırdemir ${ }^{2}$ and Barbaros Baykal ${ }^{2}$ \\ ${ }^{1}$ Agrl State Hospital, Orthopedics and Traumatology Agrl, Turkey \\ ${ }^{2}$ Departments of Orthopedics and Traumatology, Suleyman Demirel University, Turkey
}

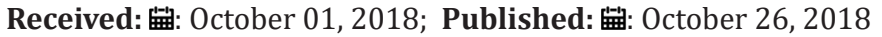

*Corresponding author: Özgür Başal, Ministry of Health, Ağrı State Hospital, Orthopedics and Traumatology Agrı, Turkey

\section{Abstract}

Objectives: The procedure for distal interlocking of intramedullary nails can be challenging problem for surgeons. Following the nail insertion, the problems associated with the location of the distal holes and correct screw placements are well known. A lot of techniques and devices have been studied to aid distal targeting, in attempts to overcome some of the associated problems. The development of the techniques and devices help to reduce radiation doses.

Methods: Forty patients who underwent surgery with closed diaphysial fractures of the femur were operated between January 2014 to December 2015 with mean age 34 (ranged between 20-48). Four different distal locking methods were compared with similar fracture patterns.

Results: Magnetic-Manual targeting group was shown significantly better operating and fluoroscopy time than hand-helped technique. Insideout distal locking method was also shown excellent result with an accuracy of $100 \%$.

Conclusion: We, orthopedic surgeons, have many risks of surgery. The radiation exposure is just one of them. Fluoroscopy- free innovations should be more easy to reach and cheap. A prospective-randomized comparison of 4 different distal locking methods were discussed in this paper.

\section{Introduction}

Nowadays intramedullary nailing of long-bone shaft fractures is generally accepted as a gold standard treatment. Gerhard Küntscher is credited with the first use of this device in 1939. Küntscher nail was tight fitting and unlocked. This is the first described nail which has some disadvantages such as rotational instability and causing bone necrosis [1]. In the past, these kind of disadvantages has limited the use of unlocked nails. Locked intramedullary nailing of the femur has showed increasing popularity in the past two decades. In early practice, the inflatable self-locking nails were used such as the Fixion (Disc-O-Tech Medical Technologies, Unimedical BIO. Tech, Torino, Italy). However, the self-locking nails do not exhibit the same torsional qualities as distally locked screws and the self-locking capabilities are not always acceptable [2]. The literature does not suggest that self-locking nail is likely to become a good alternative to distally locked nails. Owing to the locked nails, the length of the bone-implant construction is still effectively maintained because the interlocking screws prevent shortening and intramedullary nail migration.

The use of free-hand and hand-helped techniques are became popular last twenty years. Jigs mounted on the image intensifiers were also mostly used in the past. But the use of both methods rely on radiation. This technique relies heavily upon the use of the image intensifier and thus exposes the surgeon and operating team to high doses of ionizing radiation $[3,4]$. The dependence on fluoroscopic imaging for these variations of the free-hand technique makes them undesirable and is the reason why alternative approaches are welcome [2]. Another method is nail mounted guidance. This kind of devices often have been used for distal locking with nail mounted guides. This method has some unsuccessful result because of externally mounted guides cannot compensate for the deformation of the nail. Bending and rotational forces change the place of distal holes during insertion [5]. The access to insert distal locking screws precisely without needing for image intensification has, therefore, long been the wish of orthopedic surgeons using locked nailing in the femur and tibia. In recent years, some novel methods described for the distal locking screw fixation. These new locking screw designs with a simplified and self-controlled method is reduced the radiation dose and operating time. Numerous innovative techniques have been described such as self-locking nails, navigated nails, and computer-assisted nails $[2,6]$. 
The locking procedure of IMN can, as a result, be time consuming and involve significant levels of radiation to the surgeon, and patient. All these new technological developments targets to find a new way of a radiation-free method. Because of the risk of cancer that caused by ionized radiation, some kind of dosimeters also are being used during IMN surgeries [7-10]. In this study, we compared four different methods for the distally locked nails in closed femur shaft fractures.

\section{Patients and Methods}

Forty patients (30 males, 10 female) were included to this study. All cases were operated between January 2014 to December 2015 with mean age 34 (ranged between 20-48) with closed diaphysial fractures of the femur. Four different distal locking system were applied for each 10 patients with femoral shaft fractures. These distal locking systems were Inside-Out distal locking, Hand-help guide technique, Magnetic distal locking, and Computer-assisted distal locking systems. The study was performed in a single center. All distal locking procedures were performed by a senior resident who had experienced in treating at least 5 patients with these techniques. All intramedullary nails (IMN) were fixed 2 distal and 2 proximal screws. Firstly, distal screws were fixed after IMN placement. Duration of 2 distal screw fixation, total fluoroscopy time (second) were compared for each group. All patients underwent regional anesthesia. All IMN procedures were applied in lateral decubitus position. The instrumentation was enable distal targeting by surgeons with a C-arm x-ray device (mobile C-arm, Philips BV 25 Gold, Philips Medical Systems, Netherlands). Any ionized radiation dosimeter did not located during the surgery by the surgeon or patients. One standardized set-up of the device was used for all cases. Fluoroscopy was performed for the confirmation of screw placement and screw lengths.

\section{First Group: Hand-Help Guide Technique}

Free hand or hand-helped methods are conventional techniques. Conventional techniques use $\mathrm{C}$-armed fluoroscopy to locate the distal holes. This distal locking procedure can, as a result, be time consuming and involve significant levels of radiation to the patient, and the surgeon. The passage for the screw appears as a perfect circle on the image, indicating coaxial alignment of the nail holes. An incision is made through the skin and fascia, down to the cortex at this site. A K-wire is used to determine distal holes and for penetrating medial cortex from inside the distal holes. A cannulated drill is made after K-wire placement. This technique relies heavily upon the use of the image intensifiers.

\section{Second Group: Magnetic-Manual Distal Targeting Tech- nique}

Ten patients were operated with electromagnetic guide technique (SpectruM $\mathrm{TM}_{\mathrm{TM}}$, SANATMETAL $\mathrm{TM}_{\mathrm{T}}$, Eger, Hungary). This method uses electromagnetic (EM) distal hole aiming. EM guide let surgeon to find the upper distal hole. After targeting the static hole, surgeon have to use an manual targeting device to fix this device in intramedullary nail. The manual targeting device let us to send a screw from another distal hole. At this time, a sound-check device can perform from the IMN cavity for the first screw. After first screw fixation, the manual targeting device pulls out and medial cortex of this hole drills for the second screw fixation. At the end of distal locking, we checked anteroposterior and lateral fluoroscopic images of screws with a C-armed X-ray.

\section{Third Group: Inside-Out Distal Locking Technique}

Ten patients were operated with an inside-out guide technique (DISTALOCK $_{\mathrm{TM}}$, DGIMED $_{\mathrm{TM}}$, Minnetonka, MN, USA). This method uses a drill that burrs an initial hole for the distal locking screws from inside of the intramedullary nail. A guide hook is used to ensure cable drills at optimal 90 degrees' angle. A $1.3 \mathrm{~mm}$ cable drill and $4 \mathrm{~mm}$ cannuled hook are used for the inside-out drilling through lateral cortex. This hole is then further drilled from the outside using a chase back pin and a cannuled drill. After retracting the hook, continue advancing pin through the nail. At this step, a sound-check is performed and continued drilling through far cortex. It allows faster and more accurate placement of the screws than conventional placement.

\section{Fourth Group: Computer-Assisted EM Distal Targeting Technique}

Ten patients were operated with a computer-assisted technique to intramedullary nailing (Trigen $\mathrm{TAN}_{\mathrm{TM}}$ and Trigen Metanail $_{\text {TM }}$ nails, Smith \& Nephew, Inc., Memphis, TN, USA) using the SURESHOT ${ }_{\text {тм }}$ Distal Targeting System for distal interlocking. No modifications or additional preparation were required at the setting of the operating room. This method consists of three main equipment. Computerized control unit located within the system's display unit, a hand-held "wheel-shaped" targeter that produces a focused electromagnetic field and a sensor probe, inserted in the nail and mediating information to the control unit. The system reproduces a virtual real-time image of the distal part of the nail. The surgeon sets targeter on a 3D image and mimics red and green circles on the distal hole. This technique does not require a learning curve but needs fine motor skills. Combining the visuality with the coordination of small muscles is very important to immobilize it on the display unit.

\section{Results}

There were 10 patients in each group, which were similar in age. There was no significant difference in the mean age $(34,07 \pm 8,97)$ between any groups (Table 1). All groups were analyzed by oneway ANOVA followed by Duncan's test (N 10 per group). P values $<0.05$ were considered to be statistically significant. According to the ANOVA test results, distal locking time is similar in Inside-Out and Computer-Assisted techniques. Distal locking times showed no significant difference between two groups (Group IV and III). These two methods were superior to magnetic distal locking and hand-helped guide groups. The fluoroscopy time was significantly short $(\mathrm{p}<0.05)$ in the Group IV $(2,4 \pm 0,84 \mathrm{sec})$ than group I and II but was not significant difference between "Inside-Out " group $(2,6 \pm 0,96 \mathrm{sec})$. Significant difference in use of fluoroscopy time was observed in patients who underwent Inside-out and Computerassisted techniques as compared to the other groups( $\mathrm{p}<0.05)$. Drill 
attempts $(2,9 \pm 0,73)$ was significantly difference in Group I. There was no significant difference between other three techniques. Actually, two electromagnetic targeting device (Group II and IV) were also compared in this study. This study showed that the Computed-assisted EM targeting device is superior in the use of fluoroscopy time and distal locking time than the other basic EM device (Group II).

Table 1: Comparison of each group according to the age, distal locking time, fluoroscopy time, and drill attempts.

\begin{tabular}{|c|c|c|c|c|}
\hline & $\begin{array}{c}\text { Hand-help } \\
\text { guide } \\
\text { technique }\end{array}$ & $\begin{array}{c}\text { Magnetic- } \\
\text { Manual } \\
\text { Distal } \\
\text { Locking }\end{array}$ & $\begin{array}{c}\text { Computer } \\
\text { Asisted } \\
\text { Distal } \\
\text { Locking }\end{array}$ & $\begin{array}{c}\text { Inside- } \\
\text { Out Distal } \\
\text { Locking }\end{array}$ \\
\hline $\begin{array}{c}\text { Mean Age } \\
\text { (years) }\end{array}$ & $34,5(20-46)$ & $31,2(21-8)$ & $34,5(21-47)$ & $36,1(25-48)$ \\
\hline $\begin{array}{c}\text { Mean time } \\
\text { of distal } \\
\text { locking } \\
\text { (min) }\end{array}$ & $15,1 \pm 3,44$ & $7,9 \pm 2,8$ & $4,4 \pm 1,1$ & $4,2 \pm 0,78$ \\
\hline $\begin{array}{c}\text { Fluoroscopy } \\
\text { time (sec) }\end{array}$ & $16,8 \pm 4,23$ & $6,8 \pm 1,68$ & $2,4 \pm 0,84$ & $2,6 \pm 0,96$ \\
\hline $\begin{array}{c}\text { Drill } \\
\text { attempt(for } \\
2 \text { holes) }\end{array}$ & $2,9 \pm 0,73$ & $2,4 \pm 0,51$ & $2,1 \pm 0,31$ & 2 \\
\hline
\end{tabular}

\section{Complications}

Vessel or nerve damage was not occurred during distal locking in any group. Distal screws were inserted incorrectly in three patients at first group and in one patient at second group. No incorrect screw placement was found in groups III and IV. Multiple attempts were required to locate the nail hole in groups I and II. Because of the multiple attempts, widened hole entry was detected in 3 patients in group I and 2 patients in group II. Detecting the axis of distal holes were difficult in first group. There were no new fracture or screw failure obtained during the distal locking in any group.

\section{Discussion}

Intramedullary nailing is known one of the most technically demanding procedures in orthopedic traumatology. Many technical properties and surgeon's skills determine the success of the surgery [11]. Incorrect screw placement is one of the most annoying problems for the intramedullary nailing. Rotational stability and dynamization depends on correct screw fixation. First aim of intramedullary nailing is providing a torsionally stabile and rigid fixation. This stability can achieve by the locking rigid nails. While achieving this stability, inevitable deformation of the nail after its insertion to the femur may cause incorrect distal locking [6]. Many different instrumentation has been described for the correct distal screwing. Radiation depended image identifiers are being used during past decades for a correct distal locking [12]. Ionizing radiation is harmful and potentially lethal to living beings but can have health benefits in radiation therapy for the treatment of cancer. Its most common impact is the induction of cancer with a latent period of years or decades after ionized radiation exposure. The duration of fluoroscopy time correlated with the ionized radiation dose to the hands and thyroid glands and gonads of the surgeons [13].

It has been determined by phantom measurements that the majority of radiation exposure occurred during brief exposures of the hands in the direct X-ray beam on the X-ray tube near side of the patient $[9,10]$. Müller reported the average fluoroscopy time per intramedullary nailing was $4.6 \mathrm{~min}$, the average dose of radiation to the dominant hand of the primary surgeon was $1.27 \mathrm{mSv}$ and $1.19 \mathrm{mSv}$ to the first assistant [9]. Many authors have reported the overall radiation to patient gonads. surgeon hands and thyroid glands were within acceptable limits during the different IMN methods $[7,9,10]$. Because of these reports, we did not provide any dosimeter results. On the other hand, orthopaedic surgeons are not classified radiation workers and there is no satisfactory data about cancer incidence among orthopaedic surgeons. In spite of wearing lead apron and using other preventions, stochastic (chance) effects like cancers can still occur. Recently, some radiation-free techniques have been described for distally locked nails in the literature. Selflocking systems and electromagnetic targeting devices are the most popular distal locking methods in our recent practice.

Magnetic-Manual targeting group (Group II) was shown significantly better operating and fluoroscopy time than handhelped technique (Group I). However, Computer-assisted EM targeting and the inside-out method were significant superior than group II. Negrin and Vécsei have been reported that the Sanatmetal ${ }^{\circledR}$ targeting (Magnetic-Manual targeting) device has to be considered as an appealing alternative for distal locking [11]. It can be an alternative device but our statistical results is showing the superiority of inside-out and computer-assisted techniques. In spite of the radiation-free and 3D virtuality quantities of computerassisted EM targeting, Its main disadvantage is "free-hand" philosophy of the "wheel-shaped" targeter. If you are interested in computer games, this targeter will make it very enjoyable. However, it needs some fine motor skills. In this aspect, it is a surgeon' skills depended technique, so nobody has guaranteed an accuracy of $100 \%$. Inside-out distal locking device was shown excellent result with an accuracy of $100 \%$. This technique needs a bit longer learning curve than computer-assisted EM targeting. Possible complication is drill breakage or cable bending (must be $90^{\circ}$ ). This innovative distal locking device is $100 \%$ effective on the first attempt, when compared to the other groups. Finally, the main common disadvantage of Group II, III, and IV is additional costs (disposable sensor probe [III] or EM targeter [II-IV]). From this aspect, Hand-helped distal locking technique is superior to the innovative devices.

\section{Conclusion}

Distal IMN locking is a troublesome stage that may expose surgeons, operating team and patients to a considerable dose of radiation. Numerous innovative techniques have been described for reducing radiation. Cost and availability plays an important role in the success of targeting devices, or systems, and this must also be considered. The inside-out distal locking and computer-assisted EM targeting technique have come to the forefront than conventional 
methods. Radiation-free techniques are necessary to eliminate possible risks. The inside-out guide technique (DISTALOCKTM, DGIMEDTM, Minnetonka, MN, USA), a novel radiation-free targeting system utilizing inside-out guide drilling technology for distal locking, proved to be accurate, fast and reducing operation time and radiation exposure.

\section{References}

1. Edeland H, G Eriksson (1979) Fracture of the femoral shaft and the Küntscher nail. Suggestion for a method and device for the retraction of the distal nail fragment. Acta orthopaedica Belgica 45(4): 429-433.

2. Whatling GM, LD Nokes (2006) Literature review of current techniques for the insertion of distal screws into intramedullary locking nails. Injury 37(2): 109-119.

3. Leloup T, El Kazzi W, Schuind F, Warzée N (2008) A novel technique for distal locking of intramedullary nail based on two non-constrained fluoroscopic images and navigation. IEEE Trans Med Imaging 27(9): $1202-1212$

4. Leroux T, A Khoshbin, MT Nousiainen (2015) Training Distal Locking Screw Insertion Skills to Novice Trainees: A Comparison Between Fluoroscopic- and Electromagnetic-Guided Techniques. J Orthop Trauma, 29(10): 441-446.

5. Paller DJ, Frenzen SW, Bartlett CS, Beardsley CL, Beynnon BD (2013) A three-dimensional comparison of intramedullary nail constructs for osteopenic supracondylar femur fractures. J Orthop Trauma 27(2): 9399.

\section{ISSN: 2574-1241}

DOI: $10.26717 / B J S T R .2018 .10 .001955$

Özgür Başal. Biomed J Sci \& Tech Res

(C) (P) This work is licensed under Creative

Submission Link: https://biomedres.us/submit-manuscript.php
6. Stathopoulos I, Karampinas P, Evangelopoulos DS, Lampropoulou Adamidou K, Vlamis J (2013) Radiation-free distal locking of intramedullary nails: evaluation of a new electromagnetic computerassisted guidance system. Injury 44(6): 872-875.

7. Levin PE, RW Schoen, BD Browner (1987) Radiation exposure to the surgeon during closed interlocking intramedullary nailing. J Bone Joint Surg Am 69(5): 761-766.

8. Skjeldal S, S Backe (1987) Interlocking medullary nails--radiation doses in distal targeting. Arch Orthop Trauma Surg 106(3): 179-181.

9. Muller LP, Suffner J, Wenda K, Mohr W, Rommens PM (1998) Radiation exposure to the hands and the thyroid of the surgeon during intramedullary nailing. Injury 29(6): 461-468.

10. Madan S, C Blakeway (2002) Radiation exposure to surgeon and patient in intramedullary nailing of the lower limb. Injury 33(8): 723-727.

11. Negrin LL, V Vécsei (2013) Is a Magnetic-Manual Targeting Device an Appealing Alternative for Distal Locking of Tibial Intramedullary Nails? Arch Trauma Res2(1): 16-20.

12. Karaarslan AA, Aycan H, Örgen Y, Karakaşli A, Ertem F, et al. (2016) A new femoral nail with single distal locking screw for maximum interfragment rotational stability. Acta Orthop Traumatol Turc 50(1): 89-96.

13. Mahajan A, Sumant Samuel, Atul K Saran, M K Mahajan, M K Mam (2015) Occupational radiation exposure from C arm fluoroscopy during common orthopaedic surgical procedures and its prevention. J Clin Diagn Res 9(3): 01-04.

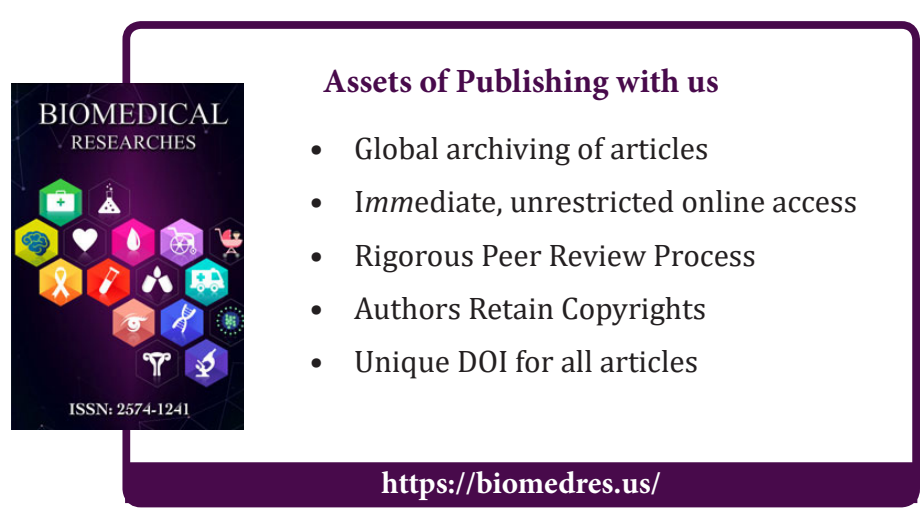

